\title{
Analysis of Stochastic Time Response of Microfluidic Biosensors in the Case of Competitive Adsorption of Two Analytes ${ }^{\dagger}$
}

\author{
Ivana Jokić ${ }^{1, *}$, Zoran Djurić ${ }^{2}$, Katarina Radulović ${ }^{1}$ and Miloš Frantlović ${ }^{1}$ \\ 1 ICTM-Institute of Microelectronic Technologies, University of Belgrade, 11000 Belgrade, Serbia; \\ kacar@nanosys.ihtm.bg.ac.rs (K.R.); frant@nanosys.ihtm.bg.ac.rs (M.F.) \\ 2 Institute of Technical Sciences of SASA, Serbian Academy of Sciences and Arts, 11000 Belgrade, Serbia; \\ zoran.djuric@itn.sanu.ac.rs \\ * Correspondence: ijokic@nanosys.ihtm.bg.ac.rs; Tel.: +381-11-2638-188 \\ † Presented at the Eurosensors 2018 Conference, Graz, Austria, 9-12 September 2018.
}

Published: 13 December 2018

\begin{abstract}
A model of stochastic time response of adsorption-based microfluidic biosensors is presented, that considers the competitive adsorption-desorption process coupled with mass transfer of two analytes. By using the model we analyze the expected value of the adsorbed particles number of each analyte, which determine the sensor response kinetics. The comparison with the case when only one analyte exists is used for investigation of the influence of competitive adsorption on the sensor response. The response kinetics analyzed by using the stochastic model is compared with the kinetics predicted by the deterministic response model. The results are useful for optimization of micro/nanosensors intended for detection of substances in ultra-low concentrations in complex samples.
\end{abstract}

Keywords: microfluidic sensor; stochastic time response; competitive adsorption; mass transfer

\section{Introduction}

Miniaturization of microfluidic adsorption-based biosensors and lower detectable concentration levels are followed by increased fluctuations of the number of adsorbed particles due to coupled random adsorption-desorption (AD) and mass transfer (MT) processes of analyte particles. The sensor response analysis thus requires the use of stochastic approach. In such sensors particularly challenging is the detection of a target substance present in a low concentration in complex samples that contain other substances that also bind to the sensing surface, affecting the sensor response.

In [1] the sensor response is analyzed by using the stochastic model that neglects the MT influence, while in [2] the analysis is based on the stochastic simulation considering AD noise and diffusion. A model that takes into account the coupling of $\mathrm{AD}$ with convection and diffusion is presented in [3]. However, neither of the published stochastic analyses of sensors time response, that take into account MT, considers the influence of competitive adsorption, which exists in practical situations of non-ideal sensor selectivity, multianalyte detection, or experiments based on such type of $\mathrm{AD}$ processes.

Here we present a model of sensor stochastic time response that considers competitive adsorption coupled with MT (both convection and diffusion) of particles of two analytes. By using the model we analyze the expected value of the adsorbed particles number of each analyte, which determine the sensor response kinetics. We investigate the influence of competitive adsorption on the stochastic sensor response and the difference between the response kinetics predicted by the stochastic and by the deterministic model. 


\section{Theory}

A stochastic model of microfluidic chemical or biological sensor response, that takes into account the coupling between $\mathrm{AD}$ and MT processes of a single analyte, is obtained by applying the master equation for gain-loss processes, and the two-compartment model (TCM [4]), in order to approximate both spatially and time dependent analyte concentration in the sensor reaction chamber [3]. The expressions for the probabilities of the effective adsorption and desorption per unit time, which model the combined effect of stochastic AD and MT processes on the change of the number of adsorbed particles, were an important step towards obtaining the model. The same approach can also be used in the case of competitive adsorption of two substances, which is treated as a bivariate gain-loss process with the states $\left(N_{1}, N_{2}\right)$, where $N_{1}$ and $N_{2}$ are random processes, representing the number of adsorbed particles of substances 1 and 2, respectively, at the moment $t$. Assuming that in the time interval $d t \rightarrow 0$ the change of the number of adsorbed particles of only one substance is possible, and at most by 1 , the transition rates between adjacent states are: $A_{1}\left(N_{1}, N_{2}\right)$, denoting the probability of increase of the number of type 1 adsorbed particles per unit time, $D_{1}\left(N_{1}\right)$ denoting the probability of decrease of $N_{1}$ by 1 per unit time, and the corresponding probabilities for $N_{2}$ are $A_{2}\left(N_{1}, N_{2}\right)$ and $D_{2}\left(N_{2}\right)$, respectively. Assuming adsorption of one particle to one binding site, uniformity of all binding sites, and the absence of interaction between analyte particles, the use of TCM for approximation of the spatial distribution of time dependent concentrations of both particle types in the reaction chamber yields theexpressions

$$
\begin{aligned}
& A_{1}=k_{a 1} \frac{C_{1}+k_{d 1} N_{1} /\left(k_{m 1} A\right)}{1+k_{a 1}\left(N_{m}-N_{1}-N_{2}\right) /\left(k_{m 1} A\right)}\left(N_{m}-N_{1}-N_{2}\right), D_{1}=k_{d 1} N_{1}, \\
& A_{2}=k_{a 2} \frac{C_{2}+k_{d 2} N_{2} /\left(k_{m 2} A\right)}{1+k_{a 2}\left(N_{m}-N_{1}-N_{2}\right) /\left(k_{m 2} A\right)}\left(N_{m}-N_{1}-N_{2}\right), \quad D_{2}=k_{d 2} N_{2},
\end{aligned}
$$

where $C_{1}$ and $C_{2}$ are concentrations of two substances in the analyzed sample, $k_{a 1}$ and $k_{a 2}$ are their adsorption rate constants, $k_{d 1}$ and $k_{d 2}$ are desorption rate constants, $k_{m 1}$ and $k_{m 2}$ are mass transfer coefficients (they model particle transfer by both convection and diffusion between the bulk solution and binding sites, according to TCM), and $N_{m}$ is the total number of binding sites on the sensing surface.

Starting from the definitions of expected values of the numbers of adsorbed particles of two substances, $\left\langle N_{1}>\right.$ and $\left\langle N_{2}\right\rangle$, their variances, $\sigma_{1}{ }^{2}$ and $\sigma_{2}{ }^{2}$, and the covariance, $\sigma_{12}$, and by using the master equation for the probability of states of the bivariate random process, the system of five equations is obtained for the mentioned first and second moments (the nonlinear transition rates (Equations (1) and (2)) are approximated by the Taylor series; all derivatives are calculated for $N_{1}=\left\langle N_{1}\right\rangle$ and $N_{2}=$ $<N_{2}>$ )

$$
\begin{gathered}
\frac{d}{d t}<N_{1}>\approx A_{1}-D_{1}+\frac{1}{2}\left[\frac{\partial^{2} A_{1}}{\partial N_{1}^{2}} \sigma_{1}^{2}+2 \frac{\partial^{2} A_{1}}{\partial N_{1} \partial N_{2}} \sigma_{12}+\frac{\partial^{2} A_{1}}{\partial N_{2}^{2}} \sigma_{2}^{2}\right], \\
\frac{d}{d t}<N_{2}>\approx A_{2}-D_{2}+\frac{1}{2}\left[\frac{\partial^{2} A_{2}}{\partial N_{1}^{2}} \sigma_{1}^{2}+2 \frac{\partial^{2} A_{2}}{\partial N_{1} \partial N_{2}} \sigma_{12}+\frac{\partial^{2} A_{2}}{\partial N_{2}^{2}} \sigma_{2}^{2}\right], \\
\frac{d \sigma_{1}^{2}}{d t} \approx A_{1}+D_{1}+\left[2\left(\frac{\partial A_{1}}{\partial N_{1}}-\frac{\partial D_{1}}{\partial N_{1}}\right)+\frac{1}{2} \frac{\partial^{2} A_{1}}{\partial N_{1}^{2}}\right] \sigma_{1}^{2}+\left(2 \frac{\partial A_{1}}{\partial N_{2}}+\frac{\partial^{2} A_{1}}{\partial N_{1} \partial N_{2}}\right) \sigma_{12}+\frac{1}{2} \frac{\partial^{2} A_{1}}{\partial N_{2}^{2}} \sigma_{2}^{2}, \\
\frac{d \sigma_{2}^{2}}{d t} \approx A_{2}+D_{2}+\frac{1}{2} \frac{\partial^{2} A_{2}}{\partial N_{1}^{2}} \sigma_{1}^{2}+\left(2 \frac{\partial A_{2}}{\partial N_{1}}+\frac{\partial^{2} A_{2}}{\partial N_{1} \partial N_{2}}\right) \sigma_{12}+\left[2\left(\frac{\partial A_{2}}{\partial N_{2}}-\frac{\partial D_{2}}{\partial N_{2}}\right)+\frac{1}{2} \frac{\partial^{2} A_{2}}{\partial N_{2}^{2}}\right] \sigma_{2}^{2}, \\
\frac{d \sigma_{12}}{d t} \approx \frac{\partial A_{2}}{\partial N_{1}} \sigma_{1}^{2}+\left(\frac{\partial A_{1}}{\partial N_{1}}-\frac{\partial D_{1}}{\partial N_{1}}+\frac{\partial A_{2}}{\partial N_{2}}-\frac{\partial D_{2}}{\partial N_{2}}\right) \sigma_{12}+\frac{\partial A_{1}}{\partial N_{2}} \sigma_{2}^{2} .
\end{gathered}
$$


By using this system of equations the stochastic time response of microfluidic biosensors in the case of competitive adsorption of two analytes can be analyzed, since it equals

$$
r(t)=m_{1} N_{1}(t)+m_{2} N_{2}(t)
$$

( $m_{1}$ and $m_{2}$ are the weight factors, which represent the average contribution of a single particle of the first and second analyte to the sensor response).

\section{Results and Discussion}

We analyze the stochastic time response of a microfluidic biosensor with two proteins being adsorbed on its active surface. The response is analyzed through the numbers of adsorbed particles of both types, which determine the sensor response (Equation (8)). Figure 1a shows the time dependent expected value of the adsorbed particles number of both target (index " 1 ") and competitor (index " 2 ") analyte, obtained by using the presented model, for the parameter values: $C_{1}=1 \mathrm{nM}, k_{a 1}=$ $8 \times 10^{7} 1 /(\mathrm{Ms}), k_{d 1}=0.081 / \mathrm{s}, k_{m 1}=2 \times 10^{-5} \mathrm{~m} / \mathrm{s} \mathrm{C} C_{2}=2 \mathrm{nM}, k_{a 2}=8 \times 10^{6} 1 /(\mathrm{Ms}), k_{d 2}=0.021 / \mathrm{s}, k_{m 2}=2 \times 10^{-5}$ $\mathrm{m} / \mathrm{s}, A=1 \times 10^{-9} \mathrm{~m}^{2}$, and the adsorption sites surface density $n_{m}=N_{m} / A=1 \times 10^{-11} \mathrm{Mm}\left(1 \mathrm{M}=1 \mathrm{~mol} / \mathrm{dm}^{3}\right)$. Figure $1 \mathrm{~b}$ shows the expected number of adsorbed particles when only the target analyte exists in the analyzed sample with the same concentration $C_{1}$ (the stochastic response model for a single analyte is presented in [3]). The influence of competitive adsorption on the change of the number of adsorbed target analyte particles is pronounced, and it is quantitatively determined based on the shown diagrams.

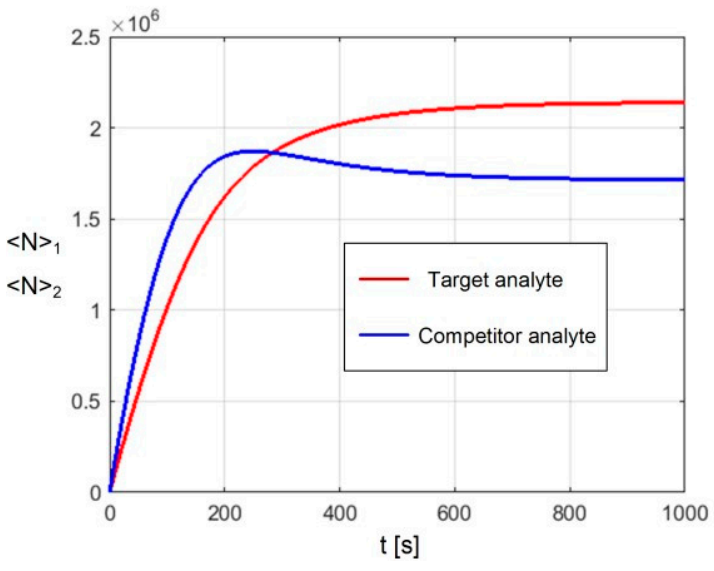

(a)

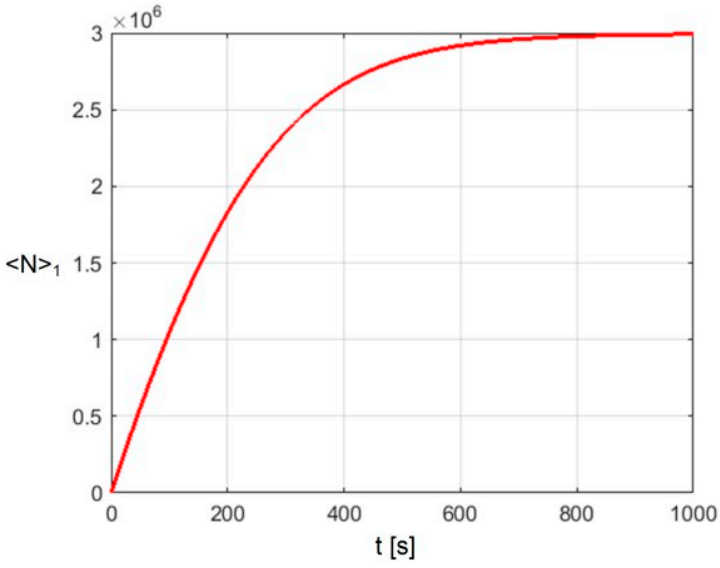

(b)

Figure 1. (a) Time dependent expected value of the adsorbed particles number of the target (red line) and the competitor analyte (blue line), obtained by using the stochastic response model; (b) Expected number of adsorbed particles when only the target analyte is present in the analyzed sample.

The time evolution of the expected value of the numbers of adsorbed particles of two analytes, which determines the sensor response kinetics, is shown in Figure 2 (solid lines), while the numbers of adsorbed particles according to the deterministic model of sensor response $\left(N_{D 1}, N_{D 2}\right)$, which takes into account mass transfer [5], are denoted by dashed lines, for a sensing area $A=1 \times 10^{-14} \mathrm{~m}^{2}$ with $N_{m}$ $=6000$ binding sites, $C_{1}=50 \mathrm{pM}, C_{2}=100 \mathrm{pM}$, and the same values of other parameters as for Figure 1. A significant difference between the kinetics predicted by the deterministic model and by the more accurate stochastic model of sensor response can be seen. It is interesting to note that the two models give an opposite prediction regarding the analyte that is dominantly adsorbed. 


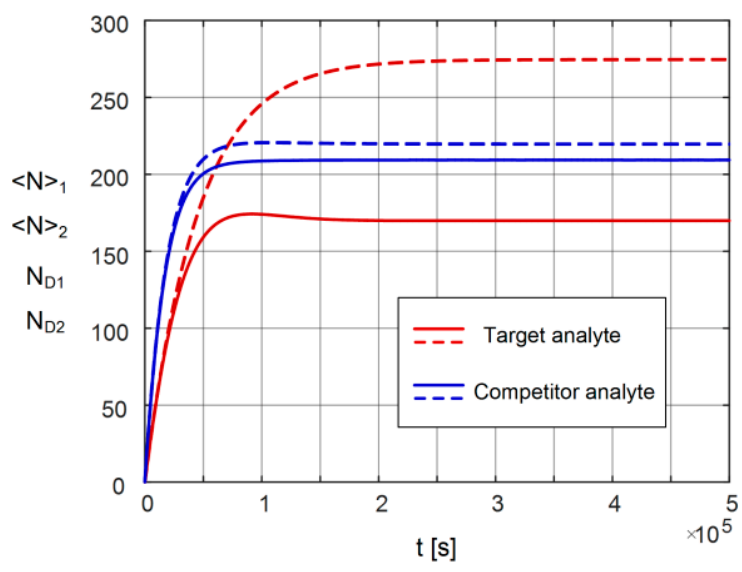

Figure 2. Time evolution of the expected value of the adsorbed particles numbers of two analytes, obtained by using the stochastic model of sensor response (solid lines), and the time dependence of the numbers of adsorbed particles according to the deterministic model of sensor response (dashed lines).

\section{Conclusions}

We presented the stochastic model of time response of a sensor whose active surface is subjected to two-analyte competitive adsorption influenced by mass transfer processes. The model is used for the stochastic time response analysis of a microfluidic biosensor, when two proteins compete for the binding sites on the sensing surface.

Results obtained by using the presented model enabled the quantitative analysis of the influence of competitive adsorption on the expected number of adsorbed particles of the target analyte. They also show how much the time evolution of the numbers of adsorbed particles predicted by the stochastic model differs from that obtained by using the deterministic model. It was observed that the two models can produce contradictory information regarding the dominantly adsorbed analyte, especially in cases where analyte concentration is low, and the sensing surface is of near-nanoscale dimensions.

The presented model is useful for interpretation of measurement results, and for optimization of sensors for reliable detection of substances present in ultra-low concentrations.

Author Contributions: I.J. conceived the paper; I.J. and Z.D. developed the stochastic model of sensor response; K.R. performed the numerical calculations; I.J. and M.F. performed the analysis of the results; I.J. and M.F. wrote the paper.

Acknowledgments: This work has been funded by the Serbian Ministry of Education, Science and Technological Development (Project TR32008) and by the Serbian Academy of Sciences and Arts (Project F-150).

Conflicts of Interest: The authors declare no conflict of interest. The founding sponsors had no role in the design of the study; in the collection, analyses, or interpretation of data; in the writing of the manuscript, and in the decision to publish the results.

\section{References}

1. Jakšić, O.; Jakšić, Z.; Čupić, Ž; Randjelović, D; Kolar-Anić, L. Fluctuations in transient response of adsorption-based plasmonic sensors. Sens. Actuators B Chem. 2014, 190, 419-428, doi:10.1016/j.snb.2013.08.084.

2 Tulzer, G.; Heitzinger, C. Fluctuations due to association and dissociation processes at nanowire-biosensor surfaces and their optimal design. Nanotechnology 2015, 26, 025502, doi:10.1088/0957-4484/26/2/025502.

3. Djurić, Z.; Jokić, I; Milovanović, G. Signal-to-noise ratio in adsorption-based microfluidic bio/chemical sensors. Procedia Eng. 2016, 168, 642-645, doi:10.1016/j.proeng.2016.11.234. 
4. Myszka, D.G.; He, X.; Dembo, M.; Morton, T.A.; Goldstein, B. Extending the Range of rate constants available from BIACORE: Interpreting mass transport-influenced binding data. Biophys. J. 1998, 75, 583594, doi:10.1016/S0006-3495(98)77549-6.

5. Frantlović, M.; Jokić, I.; Djurić, Z.; Radulović, K. Analysis of the competitive adsorption and mass transfer influence on equilibrium mass fluctuations in affinity-based biosensors. Sens. Actuators B Chem. 2013, 189, 71-79, doi:10.1016/j.snb.2012.12.080.

(ㄷ) (1)

(C) 2018 by the authors. Licensee MDPI, Basel, Switzerland. This article is an open access article distributed under the terms and conditions of the Creative Commons Attribution (CC BY) license (http://creativecommons.org/licenses/by/4.0/). 